growth hereby has yet to be determined. In a skiagram taken by Dr. D. Arthur but not published of Dr. Seymour Taylor's case ${ }^{4}$ the space intervening between the heart and pericardium in the advance and retreat of the heart's action is clearly seen.

The normal amount of pericardial serum is that of lubrication. The pathological stretch of capacity of the pericardium, according to G. A. Gibson, ${ }^{5}$ has reached eight pints of serum (cardiac). According to Jackson Clarke, ${ }^{6}$ sufficient pus has been found in this viscus to mask the whole anterior pulmonary area of resonance, yet 18 ounces of blood sufficed in $m y$ case to render the pericardium taut. Such is the elasticity and capacity of the pericardium.

In spite of Dr. 'Taylor's strictures, my treatment of aneurysm medically would be rest (relaxed periodically), limited fluid, as much iodide of potassium and lactate of calcium as the patient could bear, and a prolonged stay at Woodhall, Sandrock, or Malvern-calm retreats whose spa waters are more or less rich in iodine, bromine, alum, and iron.

West Acton, W.

\section{THE PRECIPITIN REACTION IN HYDATID DISEASE.}

By D. A. WELSH, M.A., B.Sc., M.D. Edin., PROFESSOR OF PATHOLOGY IN TEE UNIVERSITY OF SYDNFY, NEW SOUTH WALES ;

$$
\text { AND }
$$

H. G. CHAPMAN, M.D., B.S. MELB.,

DEMONSTRATOR IN PHYSIOLOGY IN THE UNIVERSITY OF SYDNEY, NEW BOUTH WALES.

IN July, 1907, Fleig and Lisbonne ${ }^{1}$ announced that they had succeeded in demonstrating the existence of a specific precipitin in the blood serum of an individual affected with a hydatid cyst and in the serum of animals immunised with hydatid products. They also emphasised the diagnostic significance of the reaction. Previous work ${ }^{2}$ by Ghedini, by Joest, and by Gherardini had yielded contradictory results. With the more abundant material at our disposal we have ween able to corroborate and to supplement the main observations of Fleig and Lisbonne in regard to hydatid invasions in the human subject. We have found that when a sufficiency of blood serum is mixed with a suitable hydatid fluid and the mixture allowed to stand for 18-20 hours at the room temperature, a well-marked precipitate never fails to appear if the serum be taken from a patient infected with hydatids (nine cases), and that precipitation does not occur when the serum is taken from persons not so infected (four suspected cases and five healthy controls). In the four suspected cases examined by us a mogative reaction was obtained, and at operation in each case the absence of hydatid invasion was revealed. These results are so consistent, though as yet few in number, that sthey encourage us in the belief that in the precipitin test for hydatid invasion we have a reliable means of diagnosis. We find, however, that certain conditions are essential to the satisfactory performance of the test.

In regard to the serum (antiserum) developed in a hydatid patient, our experience has been that, with few exceptions, it is characterised by a low precipitable content. Hence, as noted by Fleig and Lisbonne, about 12 drops of the patient's serum are usually required to yield a satisfactory amount of deposit. In a previous paper ${ }^{3}$ dealing with precipitin antisera prepared in the rabbit by injection of alien blood serum or of egg-white, we noted that two drops $(0.1$ cubic centimetre) of the antiserum were usually sufficient to yield a well-marked precipitate. We were also led to the conclusions that the precipitate was derived mainly from the antiserum and that different antisera might differ greatly in their precipitable content.

Fleig and Lisbonne observed that the anti-substances in the serum of a hydatid patient may gradually disappear after operation. That this disappearance of the precipitin is not necessarily associated with removal of all the cysts is shown by one of our cases. In a man, aged 42 years, with multiple

4 The Laxcet, March 7th, 1908, p. 702

5 G. A Gibson: Textbook of Medicine. vol. ii., p 52.

1 Comptes Rendus de la Société de Biologie, tome lxii., No. 23, 1907, 29. 1198

2 Published in 1906 and quoted by Fleig and Lisbonne, loc cit. Welsh and Chapman: Proceedings of the Royal Society, B. vol. xxviii., p. 297, 1906. hydatid cysts of the liver (not all of which conld be removed at operation) we found a well-marked reaction before operation, but at the end of the sixth week and again at the end of the seventh week after operation the serum yielded only a minute trace of deposit. The patient died in the eighth week after operation, having become rapidly weaker and more emaciated, and it may be that in these circumstances his capacity for reaction was failing. On the other hand, a girl, 14 years old, with multiple hydatids of the peritoneum following a ruptured hydatid of the liver gave a well-marked reaction eight weeks after operation. She was well nourished and was leaving the hospital apparently cured. The indication, however, was that she was still infected with hydatids and that the tissues were reacting to their presence.

In regard to the hydatid fluid, our practice has been to pass it through a Ohamberland filter. We have obtained more abundant precipitates with one cubic centimetre hydatid fluid than with two cubic centimetres (as recommended by Fleig and Lisbonne), probably because the small amount of the homologous protein contained in the hydatid fluid is sufficient to produce a marked precipitation in the anti. serum, while the interaction is not hampered by over-much dilution. This also is in harmony with our previous work on serurn and egg-white precipitins.

In the course of our experiments with hydatid precipitins we encountered one hydatid fluid which gave nu precipitate, in addiments of two cabic centimetres, either with the serum of the patient from whom it was obtained, or with the serum of other hydatid patients. When, however, these tubes, after standing 48 hours without showing any precipitate, each received the further addition of one cubic centimetre of clear fluid from another hydatid invasion, distinct precipitates were obtained. It was certainly anomalous to find that the first bydatid Hluid failed to precipitate the serum of the patient from whom it was obtained, while the serum of that patient reacted strongly with the hydatid fluid obtained from a different patient. We may note that each of the hydatid fluids above referred to contained, approximately, the same amount of albumin (about 0.025 per cent.).

The fact that not all hydatid cysts contain fluid capable of interacting to give a precipitate with the serum of the patient may be an explanation of the negative results obtained by earlier workers. What we would emphasise is that hydatid fluids may vary greatly in their capacity for nteraction with the serum of a hydatid patient, but that a hydatid fluid which reacts strongly with the serum of the patient from whom it was obtained reacts also strongly with the serum of other hydatid patients. These facts are of importance in the selection of a stock of hydatid fluid in the diagnostic applications of the test.

Summary of cases of hydatid invasion, all of which gave a marked precipitate reaotion when tested before operation.1. Male, 35 years. Three small hydatid cysts with clear contents in peritoneum. Hydatid of liver removed eight years ago. 2. Male, 42 years. Multiple hydatid cysts of liver, some of which were full of clear fluid, others shrunken and inspissated. Not all cysts removed, yet reaction very feeble six and seven weeks after operation. Death in eighth week. 3. Male, eight years. Large single cyst of liver with clear contents. 4. Female, 14 years. Multiple hydatids of peritoneum with clear contents, following ruptured hydatid of liver. Marked reaction eight weeks after operation. 5. Male, 13 years. Single hydatid cyst of liver with clear fluid contents. 6. Female, 17 years. Multiple cysts of peritoneum with clear fluid contents, dense matted adhesions. 7. Male, 50 years. Suppurating hydatid cyst in muscles of thigh, well-marked reaction three weeks after operation. 8. Male, 48 years. Large hydatid cyst of liver with slightly turbid contents. 9. Male, 23 years. Large hydatid cyst of liver with slightly turbid contents.

Conclusions. - 1. The interaction between selected hydatid fluids and a sufficiency of the serum of a patient affected with hydatid disease has, in our experience of nine cases, never failed to give a positive precipitin reaction when tested before operation. 2 Not all hydatid fluids are capable of eliciting this reaction, and their failure is not associated with any noticeable diminution of their protein content. 3. Persistence of a marked reaction some weeks after operation probably indicates the continued presence of the parasite, but the disappearance of the reaction does not necessarily indicate complete removal of the cysts. 4. A positive reaction is independent of the site of the hydatid cyst (hepatic, peritoneal, muscular) and independent also of the nature of its contents (clear, turbid, or purulent). 